\title{
Life Stress and the Long-Term Treatment Course of Recurrent Depression: III. Nonsevere Life Events Predict Recurrence for Medicated Patients Over 3 Years
}

\author{
Scott M. Monroe, Leandro D. Torres, \\ and Julien Guillaumot \\ University of Oregon \\ John E. Roberts \\ University of Buffalo, State University of New York
}

\author{
Kate L. Harkness \\ Queen's University
}

\begin{abstract}
Research has consistently documented the significance of severe life events for onset of major depression. Theory, however, suggests other forms of stress are relevant for depression's recurrence. Nonsevere life events were tested in relation to depression for 126 patients with recurrent depression in a 3-year randomized maintenance protocol. Life stress was assessed every 12 weeks and rated along dimensions of severity, focus, and independence. A significant interaction between specific types of nonsevere life events and medication was found. For medicated patients, subject-focused independent nonsevere life events predicted recurrence; for unmedicated patients, these events predicted fewer recurrences. Other nonsevere life events did not predict recurrence. The findings underscore the potential importance of specific stressors for triggering recurrences of depression.
\end{abstract}

Keywords: life events, depression, recurrence, treatment

It is becoming increasingly recognized that depression often runs a chronic, intermittent lifelong course (Frank \& Kupfer, 2003; Keller, 2003). According to The Diagnostic and Statistical Manual of Mental Disorders (4th ed., text rev.; DSM-IV-TR; American Psychiatric Association, 2000), at least $60 \%$ of individuals who have had one depressive episode will have another, $70 \%$ of individuals who have had two depressive episodes will have a third, and $90 \%$ of individuals with three episodes will have a fourth episode. Other upper-bound estimates suggest that over $75 \%$ of

Scott M. Monroe, Leandro D. Torres, and Julien Guillaumot, Department of Psychology, University of Oregon; Kate L. Harkness, Department of Psychology, Queen's University, Kingston, Ontario, Canada; John E. Roberts, Department of Psychology, University of Buffalo, State University of New York; Ellen Frank and David Kupfer, Department of Psychiatry, Western Psychiatric Institute and Clinic, University of Pittsburgh.

This work was sponsored in part by National Institute of Mental Health Research Grants MH39139 and MH60802 (Scott M. Monroe) and MH29618-13 (Ellen Frank). We thank Joan Anderson for participating in stress ratings, performing data entry, and data management; Greg Garamoni for conducting patient interviews; Karyn Angell, Sheri Johnson, Yvonne Johnson, Scott Fischer, Brian Goff, Shari Gwillam, John Kelley, David Lochridge, Diane Spangler, Anne Simons, Phillip Sullins, and Shari Wade for participating in stress ratings; Beth Zboran and Vicky Grochochinski for assistance with data management; and John McQuaid for helpful discussions, participation in stress ratings, and assistance with the data.

Correspondence concerning this article should be addressed to Scott M. Monroe, who is now at the Department of Psychology, 118 Haggar Hall, University of Notre Dame, Notre Dame, IN 46556, E-mail: smonroe1@nd.edu depressed patients have more than one depressive episode (Gotlib \& Hammen, 2002; Keller \& Borland, 1998). If one includes related subsyndromal conditions, the extent of the long-term morbidity is yet greater (e.g., Angst, Kasper, \& Weiller, 2000). Despite the modern era of pharmaceutical intervention, the most recent data suggest that the frequency of recurrent episodes has not changed (Kanai et al., 2003; Kennedy, Abbott, \& Paykel, 2003; Lee, 2003). A key consideration for understanding the lifelong impact of mood disorders consequently concerns factors that influence recurrence and the long-term clinical course of depression.

Although much has been learned over the past 2 decades about the descriptive epidemiology of recurrent depression, comparatively little has been learned about the processes involved with the initiation of recurrent episodes. Few data exist to help explain (a) why some people with depression later experience recurrences although others do not, or (b) why those who experience a recurrence break down at any particular time. In this light, one of the more promising avenues of inquiry involves life stress. A large research literature consistently has documented the importance of major life stress for the onset of a depressive episode (Brown \& Harris, 1989; Hammen, 2005; Mazure, 1998; Monroe \& Simons, 1991)

With particular reference to the recurrence of depression, however, stress is considered to play a more complicated and nuanced role. On the basis of research suggesting that severe, major life stress is more common prior to onset of first versus subsequent episodes of depression, Post (1992) proposed the "kindling" or "sensitization" hypothesis. The basic premise of this hypothesis is that while major stress is important for precipitating a first lifetime 
episode of depression, major life stress becomes progressively less uniquely critical for precipitating later recurrences. There is general agreement in the literature on this point: major stress figures most prominently for episodes early in the lifetime course of the disorder. Yet there is also considerable confusion about the changing role of life stress over successive recurrences (Hlastala et al., 2000; Monroe \& Hadjiyannakis, 2002). Much of the confusion stems from whether or not recurrences of depression can eventually (a) be brought about by progressively less severe stressors or (b) become autonomous of, or uncorrelated with, life stress. A key conceptual issue, then, concerns whether or not nonsevere forms of life stress become capable of triggering recurrences of depression (see Monroe \& Harkness, 2005).

Given a theoretical emphasis on life stress for the recurrence of depression, it is surprising that very little research to date has examined the role of nonsevere life stress for predicting depression's long-term course. Ormel, Oldehinkel, and Brilman (2001) provided initial support for such ideas, where they found that mild stressful life events predicted a new episode of subsyndromal or major depression, but only for individuals with a prior history of depression (subsyndromal or major depression). Since the depressed group was an elderly community sample that was predominantly subsyndromal (69.9\%), however, the implications for major depression and its recurrence in general adult populations remain unclear.

Consonant with the findings of Ormel et al. (2001), Monroe, Roberts, Kupfer, and Frank (1996) found that undesirable life events prospectively predicted a new episode of depression for recurrent depressives receiving maintenance medication treatment. Interestingly, life stress did not predict the onset of a new episode for recurrent depressives without maintenance medication. The results from Monroe et al. (1996) are congruent with the kindling framework and suggest an important role of nonsevere events in bringing about recurrences of depression. Because patients were carefully selected to have a high likelihood of recurrence (e.g., three or more prior lifetime episodes, with an average of over six lifetime episodes per patient), the diathesis for depression was very strong. Without medication, recurrence is a very frequent outcome for such individuals (i.e., 67\%; Frank et al., 1990). Recurrence has become either autonomous of social stressors, or so exquisitely sensitive to minor social perturbations, that even relatively minor life events may not be needed to bring about a recurrence (Kendler, Thornton, \& Gardner, 2000; Monroe \& Harkness, 2005; Post, 1992).

Viewed in this light, medication counteracts the accruing and eventually extreme neurobiological stress sensitivity, thereby lessening the person's extreme susceptibility. As a consequence, more impactful stressors are once again needed to initiate a new recurrence. In a sense, medication can be viewed as reversing the neurobiologic progression of sensitization, bringing back into predictive play stressors of a moderate, but not necessarily severe, magnitude. Interestingly, similar ideas have been advanced to account for parallel findings in research on medicated and unmedicated schizophrenic patients and relapse, where life events also were found to be predictive of relapse for the medicated, but not unmedicated, patients (Leff, Hirsch, Gaind, Rohde, \& Stevens, 1973). Given the overarching importance of continued medication treatment for the prevention of recurrence in recurrent depressed populations (Frank et al., 1990), its role with regard to life stress over the lifetime course of recurrent depression represents an important yet understudied issue.

A major obstacle investigators face in studying the potential role of nonsevere life events in depression's recurrence is the tremendous heterogeneity of experiences typically included within this broad category. Severe life events are relatively uniform in terms of their high degree of threat and clear psychological importance for the person. In contrast, nonsevere life events are much more varied in terms of their threat value and likely psychological meaning. Many, if not most, nonsevere events are also probably quite mixed in their potential to trigger depression's recurrence. To more fully understand the role of life stress in the recurrence of depression, it would be productive to constrain such heterogeneity and to target specific types of nonsevere life events that, in theory, are most capable of triggering a recurrence (Monroe \& Harkness, 2005).

Three considerations may prove useful for researchers seeking to document the potential of nonsevere life events to predict the recurrence of depression. First, severe events by definition involve the respondent in a direct way. The impact of the event falls squarely on the subject, as opposed to falling largely on someone else (such as a spouse, child, or more remote relatives or friends). However, many nonsevere events have a different "focus," impacting primarily others within the subject's social field. For example, a sister's marital or health problems, a spouse's work events, or a child's minor illness might be reported, but all these experiences represent events that likely have little direct impact on, or direct meaning for, the subject. As one descends the gradient of event severities, more events are included that are likely to be "other-focused" (i.e., circumstances that only peripherally involve the subject). Distinguishing nonsevere life events with regard to focus thus represents one promising direction for studying factors leading to the recurrence of depression.

The second aspect of life stress that could influence the impact of life events is independence, or the degree to which events are beyond the control of the individual. Studies on life stress and depression have reported that major events that are outside of the control of the individual may be especially potent for predicting onset of depression ("independent" or "fateful" life events; see Shrout et al., 1989; Stueve, Dohrenwend, \& Skodol, 1999). These findings are reinforced by large literatures on both human and animal models of depression that indicate a lack of control over stressors increases stress responses and predicts depressive reactions (Abramson, Seligman, \& Teasdale, 1978, Anisman \& Matherson, 2005; Maier \& Watkins, 2005). Certainly many nonindependent types of major life events also predict depression (e.g., dismissal from work, relationship breakup); indeed, severe life events in general (independent and nonindependent) represent one of the most robust predictors of depression's onset (see Monroe \& Hadjiyannakis, 2002). For research testing the changing role of life stress over time in the recurrence of depression, however, we again underscore the need to delimit the broad and heterogeneous class of nonsevere life events and to concentrate upon nonsevere events with suspected psychological importance. At the present stage of knowledge, it is also useful to differentiate nonsevere life events with regard to the independence of life events.

The third issue for evaluating the potential role of nonsevere life events pertains to assessment considerations. There is general agreement in the life stress literature that self-report methods 
suffer from several drawbacks (e.g., Kessler, 1997), and that interview-based assessments using contextual methods are preferred (Hammen, 2005). These latter procedures are typically based on semistructured interviews, wherein both the occurrence of life events is systematically probed and detailed biographical information is elicited about the circumstances of the person's life. Such information provides both the requisite information to determine if an experience meets operational criteria for defining a life event, and to determine the likely meaning of the event given the person's particular biographical context (Brown \& Harris, 1989). Such contextual information, too, is essential for evaluating the focus and independence of life events. Overall, employing interview and contextually based methods to differentiate nonsevere life events (a) by focus and (b) by independence enhances the potential for detecting the elusive relations between less severe degrees of life stress and the recurrence of depression.

The purpose of the present study is to provide prospective findings for nonsevere life stress in relation to the recurrence of depression over a 3-year period of maintenance treatment for individuals with recurrent depression. The participants represent an expanded and final sample from previous reports on (a) life stress and acute treatment response in recurrent depression (Monroe, Kupfer, \& Frank, 1992) and (b) life stress and recurrence of depression (Monroe et al., 1996). ${ }^{1}$ In accord with the kindling hypothesis and psychological theory, we predicted that nonsevere life events that are subject focused and "fateful" (i.e., independent) would be the strongest predictors of recurrence (Monroe \& Harkness, 2005; Post, 1992). In addition, on the basis of the highly recurrent nature of our sample and the role of medication in lowering susceptibility to recurrence, we hypothesized that patients receiving active medication would be most likely to suffer a recurrence following a nonsevere life event (Monroe et al., 1996).

\section{Method}

\section{Overview}

The general design of the research covers three distinct phases, with the first two phases ensuring an appropriate sample for studying recurrence of major depression. The third phase is the time period of primary interest for the present study. The first phase is the Acute Phase, during which depressed patients received a combined treatment of imipramine (150-300 $\mathrm{mg}$ ) and interpersonal psychotherapy (IPT; Klerman, Weissman, Rounsaville, \& Chevron, 1984). The second phase is the Continuation Phase, a 17 -week period immediately following a favorable clinical response to the Acute Phase treatment; during this phase patients continued to receive the same combined treatment. To remain in the protocol during this Continuation Phase, patients were required to sustain symptom improvement over the 17-week duration (see below).

The third phase is the Maintenance Phase, which involves a 3-year period following successful completion of the Continuation Phase (i.e., remission). In the Maintenance Phase patients were randomized into different treatment protocols (see below). Life stress was assessed continuously during the Maintenance Phase, beginning at entry into the Maintenance Phase for the prior 12 weeks and continuing every 12 weeks until recurrence or termination from the study. The present focus is on life stress and recurrence of depression over this 3-year period. Overall, it is important to recognize that the research was designed specifically to study factors predictive of the recurrence of depression (i.e., recurrence cannot be studied without prior recovery that is complete and sustained).

\section{Participants}

Participants were recruited through self-referral, medical referral, and a public information campaign for treatment of recurrent depression (see Frank et al., 1990; Monroe et al., 1996). All subjects were required to (a) be in at least their third episode of definite major depression $(M=6.84$ prior episodes in the present sample) as determined by Research Diagnostic Criteria (RDC; Spitzer, Endicott, \& Robins, 1978); (b) be between the ages of 21 and 65 years; (c) have had no more than $2 \frac{1}{2}$ years between the onset of the index episode and the most recent previous episode; and (d) have had at least a 10-week period of remission separating the previous episode from the index episode. Patients were also required to score 7 or above on the Raskin Severity of Depression Scale (Raskin, Schulterbrandt, Reatig, \& McKeon, 1969) and 15 or above on the Hamilton Rating Scale for Depression (HRSD, 17-item version; Hamilton, 1960) on two evaluations separated by 2 weeks. Exclusion criteria included medical conditions (pregnancy; major cardiovascular, renal, liver, or endocrine disease; organic brain syndrome; mental retardation; or a medical history precluding treatment with tricyclic antidepressants) and other psychiatric conditions (schizophrenia, schizoaffective disorder, unspecified functional psychosis, alcohol abuse, and drug abuse).

We selected 126 consecutive admissions to the maintenance phase of this study on recurrent depression for participation in the present report (see Frank et al., 1990). ${ }^{2}$ Mean duration of the index episode prior to treatment entry for these 126 remitting subjects was 22.4 weeks, and mean age at protocol entry was 40.14 years $(S D=10.98)$. Of the subjects, $97.0 \%$ were White, $52.4 \%$ were currently married and not separated, and $76.2 \%$ were female. Participants were informed about the study shortly after they began treatment at Western Psychiatric Institute and Clinic, and on agreeing to participate in the research, completed written informed consent prior to the first life stress interview. The research protocol was reviewed and approved by the Institutional Review Board of the University of Pittsburgh

\section{Materials}

Patients meeting criteria for entry into the treatment protocol were administered a comprehensive battery of assessments. The measures of relevance for the present report are life stress, depression, the definition of treatment response, and the definition of recurrence.

Life stress assessment. Study participants received at each assessment a modified version of the Psychiatric Epidemiology Life Events Research Interview (PERI) Life Events Scale (Dohrenwend, Krasnoff, Askenasy, \& Dohrenwend, 1978). The PERI Life Events Scale is a self-report checklist developed in response to criticisms of the first generation life events checklists (e.g., the Schedule of Recent Experiences; Holmes \& Rahe, 1967); our modified version contained 110 events (including provisions for

\footnotetext{
${ }^{1}$ In these prior reports, we found that negative life events occurring earlier in the course of the treatment protocol prospectively predicted treatment response, postrecovery symptom course, and recurrence of depression. Although we had earlier examined life stress and recurrence for the same 3-year period as in the present study $(n=53)$, the present study is based on the final study sample $(N=126)$. In addition, the past work was based on only one life stress assessment covering a single 12-week period prior to beginning the 3-year period. For the present study, we reported on life stress assessed more sensitively with longitudinal interviews conducted every 12 weeks over the 3-year maintenance treatment period.

${ }^{2}$ We note that 2 patients who entered the study at the very beginning were not available for life stress interviews prior to developing a recurrence of depression. Consequently, instead of a sample of 128 patients as reported in the original treatment study (see Frank et al., 1990), the present research is based on a sample of 126 patients.
} 
writing in events not covered in the original). Following completion of the PERI Life Events Scale, each person was administered a semistructured interview in which the endorsed events were probed with specific questions tailored to the particular event. Further information also was sought concerning other aspects of the individual's life to provide biographic details required to perform the subsequent ratings (described below). All interviews were tape-recorded.

Life stress assessments were scheduled every 12 weeks beginning with entry into the Maintenance Phase. Participants were instructed to report all events occurring within 12 weeks prior to the day of the interview. At each subsequent 12-week assessment, participants were instructed to report all intervening events from the date of the prior interview. Exact dates for the prior assessment were always provided, and the interviewer routinely had possession of the prior life stress assessment (to ensure that the timing was correct, to follow up on prior reported stressors, and to avoid reporting events from the prior time period in the current time period; McQuaid, Monroe, Roberts, \& Johnson, 1992). Life stress assessments continued throughout the patient's involvement until (a) recurrence $(n=61)$, (b) early termination from the study (i.e., attrition; $n=22$ ), or (c) completion of the 3-year protocol $(n=43)$.

When participants terminated the protocol early or suffered a recurrence (see following definition of recurrence), they received an immediate and final life stress assessment (typically earlier than the scheduled 12-week assessment). For predicting recurrence, the last life event assessment period for all patients was used. This assessment was based on the date of the last interview for patients without recurrence, or on the date of recurrence onset for those with a recurrence. Because study participants visited the clinic on a monthly basis, relatively fine-grained symptom data were available to help determine the timing of mood changes and to ensure that life events preceded onset of the recurrence episode (see also below).

The information from the life stress interview was presented to a panel of raters trained in the Bedford College Life Events and Difficulties Schedule (LEDS) procedures. ${ }^{3}$ For all of these tasks, the LEDS manuals were available to provide anchoring examples and standardization (e.g., there are approximately 5,000 case vignettes to assist in defining events and assigning threat ratings). Raters were blind to information about the person's subjective response to stressors and to the person's clinical status (i.e., depressive symptoms or recurrence status). All events that could be direct consequences of depression ("dependent" events) were excluded from the analyses by ensuring events occurred prior to the established timing of onset and were not part of an insidious prodrome (e.g., work problems due to poor concentration).

Within the LEDS system, severe events are defined as events rated very high on long-term threat (i.e., essentially a 1 or 2 on a 5-point scale) that directly affect the subject (i.e., are subject or joint focused; Brown \& Harris, 1978). For investigating the recurrence of depression, however, we have emphasized the strong theoretical grounds for considering that nonsevere life events play an important role in initiating recurrence onset (Hlastala et al., 2000; Monroe \& Harkness, 2005; Post, 1992). Nonsevere events are more common and less threatening (i.e., are rated 3 to 5 on the 5-point scale of long-term threat), and can involve the subject or primarily important others in the subject's social field. To limit the heterogeneity of the nonsevere events and to target events that likely possess the most psychological meaning, we used both focus and independence ratings of events to construct categories of event exposure to nonsevere events. (Both focus and independence are routine ratings within the LEDS system.) All life events were rated on focus as (a) "subject" or (b) "any" focus ("any" focus included "subject" focus), and on independence as (a) "independent" or (b) "independent" and "possibly independent" ("possibly independent" included "independent" events). ${ }^{4}$ Note that "possibly independent" events within the LEDS approach refer to events that are frequently but not necessarily a result of the respondent's behavior (e.g., loss of employment, relationship breakdown). (In contrast, "dependent" events within the LEDS system are events that are due to depressive functioning.)
Within the LEDS rating system, life stress is rated on the basis of extensive information about the circumstances surrounding the event and on the particular individual's biographic circumstances (i.e., "contextual" ratings; see Brown \& Harris, 1978, 1989). There is no singular assignment of scores based on summary descriptions of the events. Nonetheless, examples are useful to portray the types of events typically included for the range of severities in the present study. Examples of severe events include termination of a core relationship, a broken engagement, and a very serious fight with spouse. Examples of nonsevere events include appearing for a court case, greatly reduced contact with sister, termination from a part-time writing job, moving, start of new job, and beginning a likely problematic romantic relationship. Examples of independent subject-focused events include spouse's loss of job (with significant loss of family income) and loss of subject's employment due to company closing. Overall, the LEDS system has proved to be a very reliable and valid measure of life stress (see Brown \& Harris, 1989; Monroe \& Roberts, 1990). In a previous project using the same procedures, pairwise comparisons of four raters on longterm threat ratings ranged from .76 to $.81(M=.78$; corrected for chance agreement with Cohen's kappa).

Symptom measures. The 17-item HRSD (Hamilton, 1960), the Raskin Severity of Depression Scale (Raskin et al., 1969), and the 21-item Beck Depression Inventory (BDI; Beck, Steer, \& Garbin, 1988) were used to assess the presence and severity of depressive symptoms at each clinic visit. The HRSD and Raskin scales were completed by a clinical evaluator; the BDI was completed by the patient. Clinicians performing the HRSD were blind to the patient's stress ratings. The Raskin Severity of Depression Scale is a commonly used measure to assess depressive functioning in patient populations and was used in the present study in concert with the HRSD for patient selection and for defining clinical response (see following paragraph). All measures possess well-documented reliability and validity for measuring depressive symptoms (Rabkin \& Klein, 1987).

Definition of clinical responses and recurrence. Patients initially were required in the Acute Phase to attain scores of $\leq 7$ on the HRSD and $\leq 5$ on the Raskin scale for 3 consecutive weeks to be considered treatment responders. Once an individual responded to treatment, he or she was then required to maintain the response criteria (i.e., $\leq 7$ on the HRSD and $\leq 5$ on the Raskin scale) for an additional 17 weeks in the Continuation Phase. After successful completion of this phase, patients entered the Maintenance Phase and were randomly assigned to one of 5 treatment conditions. Patients received the assigned maintenance treatment until they (a) left the protocol (attrition owing to moving, noncompliance), (b) met formal RDC diagnosis of recurrence of depression, or (c) completed the full 3 years in the maintenance treatment protocol.

Recurrence of depression was defined formally by a two-step procedure. If a patient presented with substantial symptoms, he or she was observed and evaluated twice within a 7-day period. If an independent evaluator and the patient's clinician judged that the patient met RDC for major depressive disorder and the independent evaluator rated the patient as having a minimum of HRSD $\geq 15$ and Raskin $\geq 7$ on both occasions, the patient was seen by an independent senior psychiatrist. The latter person was not

\footnotetext{
${ }^{3}$ Raters were trained by Scott M. Monroe, who was trained in the LEDS procedures by Tirril Harris. The number of raters per case ranged from 1 to 4 , with the vast majority involving at least two raters.

${ }^{4}$ The two focus categories were overlapping, as were the two independence categories. For example, subject-focused events were included within the broader class of any-focus events. In a similar manner, independent events were included within the broader category of "independent and possibly independent" events. Theoretically, there are sound reasons for considering subject-focused or independent events separately. The basis for considering any-focus except subject-focused events, or entirely nonindependent events (i.e., excluding independent events), is not apparent and, therefore, was not included.
} 
affiliated with the study and was blind to the patient's treatment assignment. If the outcome of this latter evaluation indicated an episode of major depression, recurrence was formally declared.

Treatment considerations. All patients initially received the same treatment for the first two phases of the study (i.e., Acute and Continuation Phases), consisting of pharmacotherapy (imipramine, $150-300 \mathrm{mg} /$ day) and IPT (Klerman et al., 1984). Treatment sessions were scheduled weekly for the first 12 weeks, biweekly for the next 8 weeks, and then monthly. Once the patient sustained recovery criteria for a total of 20 weeks, he or she was randomized into the final phase, the 3-year maintenance treatment protocol comparing five different treatment regimens. These monthly treatments were (a) Maintenance Interpersonal Psychotherapy (IPT-M) alone $(n=26)$, (b) IPT-M with imipramine $(n=25)$, (c) IPT-M with placebo $(n=25)$, (d) imipramine and medication clinic visits $(n=28)$, and (e) placebo and medication clinic visits $(n=22$; see Frank et al., 1990).

\section{Design and Analyses}

Patients initially were divided according to the presence or absence of nonsevere life events. Nonsevere events were further distinguished by focus ("subject" vs. "any" focus) and by independence ("independent" vs. "possibly independent"). Life events occurring within a 6-week period prior to recurrence onset or to study termination were used in the analyses (this interval provides temporal sensitivity, and is in keeping with other recent reports on life stress and recurrence of depression; Kendler et al., 2000; Stueve, et al., 1999). ${ }^{5}$ Descriptive statistics for the life event data appear in Table 1. Although the focus of the study is on nonsevere events and recurrence, it should be noted that there were too few severe events to provide for additional analyses (e.g., 2 and 6 independent and possibly independent events over the 6-week interval).

The dependent variable for the 3-year follow-up was recurrence (61 patients suffered a recurrence). Four separate logistic regression analyses were conducted to test the relationship between the respective life event categories and recurrence ( $\chi^{2}$ improvement values are reported). For all primary analyses, care was taken to initially control for alternative predictors of recurrence to establish the independent relations between life stress and recurrence (see "Preliminary Analyses"). All interactions were tested in hierarchical regression analyses after prior entry of the interaction's constituent components (i.e., the main effects; Cohen \& Cohen, 1983).

Table 1

Descriptive Statistics for Categories of Nonsevere Life Events Occurring During the Prior 6-Week Period

\begin{tabular}{|c|c|c|c|c|c|}
\hline \multirow[b]{2}{*}{ Event type } & \multicolumn{2}{|c|}{$\begin{array}{l}\text { Patients } \\
\text { with } \\
\text { stress }\end{array}$} & \multicolumn{2}{|c|}{$\begin{array}{l}\text { Patients } \\
\text { without } \\
\text { stress }\end{array}$} & \multirow[b]{2}{*}{ Range $^{\mathrm{a}}$} \\
\hline & $n$ & $\%$ & $n$ & $\%$ & \\
\hline $\begin{array}{l}\text { Nonsevere events } \\
\text { Subject focus } \\
\text { Independent }\end{array}$ & 15 & 12 & 111 & 88 & $0-2$ \\
\hline $\begin{array}{l}\text { Nonsevere events } \\
\text { Subject focus } \\
\text { Possibly independent }\end{array}$ & 40 & 32 & 86 & 68 & $0-4$ \\
\hline $\begin{array}{l}\text { Nonsevere events } \\
\text { Any focus } \\
\text { Independent }\end{array}$ & 26 & 21 & 100 & 79 & $0-3$ \\
\hline $\begin{array}{l}\text { Nonsevere events } \\
\text { Any focus } \\
\text { Possibly independent }\end{array}$ & 51 & 40.5 & 75 & 59.5 & $0-5$ \\
\hline
\end{tabular}

${ }^{\text {a }}$ The range of reported events per category.
Given the number of analyses conducted, alpha was set conservatively at $p<.01$. The data were analyzed by using SPSS 11 for Macintosh OS X.

\section{Results}

\section{Preliminary Analyses}

Basic demographic and clinical variables initially were evaluated in relation to recurrence. There were no significant associations for the major demographic variables (age, marital status, sex) and recurrence. Also, there were no associations between the major clinical variables (depression history, duration of index episode before treatment entry) and recurrence, except for age of first depressive episode. Patients who suffered a recurrence had a later age of first lifetime onset $(M=29.27, S D=11.14)$ compared with patients who did not suffer a recurrence $(M=25.53, S D=$ 9.59), $t(122)=2.01, p<.05 .{ }^{6}$ For all significant findings reported subsequently, a separate analysis was run to control for these effects of age for first depression; in no instance did the inclusion of age of first depressive episode alter the reported findings.

Previously reported findings indicated that treatment was the major predictor of recurrence (Frank et al., 1990). Active medication strongly predicted a lower likelihood of recurrence, and IPT-M predicted a longer time before recurrence (Frank et al., 1990). Therefore the five treatment groups were collapsed and two dichotomous variables created: (a) presence or absence of medication and (b) presence or absence of psychotherapy. These two treatments and their interaction were routinely entered into the regression analyses prior to the life stress variables to control for treatment effects.

\section{Recurrence}

As expected, the first hierarchical step for medication and psychotherapy was a highly significant predictor of recurrence in all four regression analyses, $\chi^{2}(2, N=126)=26.02, p<.001$. None of the main effects for the respective nonsevere life event categories was significant (see Table 2). In line with our predictions, the interaction between medication status and the presence or absence of nonsevere subject-focused independent events was highly significant, $\chi^{2}(1, N=126)=12.21, p<.001 .^{8}$ In contrast, none of

\footnotetext{
${ }^{5}$ The reported findings for the 6-week intervals were compared with identical tabulations based on 12-week intervals. The results were essentially the same, with 6-week data yielding slightly more robust findings. The basic interpretation of the findings, however, was not altered by the particular timeframe adopted.

${ }^{6}$ This difference appeared to be attributable to a trend for patients on medication in the present sample to have an earlier first lifetime onset of depression, $t(122)=1.93, p<.06$.

${ }^{7}$ Preliminary analyses were also conducted for history of depression (including all main effects and interactions), with no substantive impact on the findings to be reported.

${ }^{8}$ Additional analyses were conducted predicting recurrence as a function of nonsevere events separately for medicated and unmedicated groups. For the medicated group, the presence of a nonsevere event significantly predicted recurrence, $\chi^{2}(1, N=53)=10.96, p<.001$; with continuity correction, $\chi^{2}(1, N=53)=7.99, p<.005$. For the unmedicated group, the absence of a nonsevere event predicted recurrence, $\chi^{2}(1, N=73)=5.07$, $p<.02$; with continuity correction, $\chi^{2}(1, N=53)=3.81, p<.051$.
} 
Table 2

Comparisons for Four Hierarchical Logistic Regression Models Testing Separate Nonsevere Life Event Categories and Their Interactions With Medication Status for Predicting Recurrence of Depression

\begin{tabular}{|c|c|c|c|c|c|c|}
\hline Event regression model & $\begin{array}{l}\text { Entry } \\
\text { step }\end{array}$ & Variable & $\begin{array}{c}\chi^{2} \\
\text { improvement }\end{array}$ & $B$ & $S E B$ & $p$ \\
\hline \multirow{4}{*}{$\begin{array}{l}\text { Subject focus } \\
\text { Independent }\end{array}$} & 1 & Medication & 26.02 & -2.03 & 0.43 & $<.0001$ \\
\hline & & IPT-M & & -0.34 & 0.43 & \\
\hline & 2 & Event & 0.001 & -0.02 & 0.61 & ns \\
\hline & 3 & Interaction (Event $\times$ Medication) & 12.21 & 4.37 & 1.39 & $<.001$ \\
\hline \multirow{4}{*}{$\begin{array}{l}\text { Subject focus } \\
\text { Possibly independent }\end{array}$} & 1 & Medication & 26.02 & -2.03 & 0.43 & $<.0001$ \\
\hline & & IPT-M & & -0.34 & 0.43 & \\
\hline & 2 & Event & 1.22 & -0.48 & 0.44 & $n s$ \\
\hline & 3 & Interaction (Event $\times$ Medication) & 3.56 & 1.65 & 0.88 & $<.06$ \\
\hline \multirow{4}{*}{$\begin{array}{l}\text { Any focus } \\
\text { Independent }\end{array}$} & 1 & Medication & 26.02 & -2.03 & 0.43 & $<.0001$ \\
\hline & & IPT-M & & -0.34 & 0.43 & \\
\hline & 2 & Event & 0.001 & -0.02 & 0.49 & ns \\
\hline & 3 & Interaction (Event $\times$ Medication) & 2.97 & 1.67 & 0.96 & $<.09$ \\
\hline \multirow{4}{*}{$\begin{array}{l}\text { Any focus } \\
\text { Possibly independent }\end{array}$} & 1 & Medication & 26.02 & -2.03 & 0.43 & $<.0001$ \\
\hline & & IPT-M & & -0.34 & 0.43 & \\
\hline & 2 & Event & 1.60 & -0.52 & 0.41 & $n s$ \\
\hline & 3 & Interaction (Event $\times$ Medication) & 0.85 & 0.78 & 0.85 & $n s$ \\
\hline
\end{tabular}

Note. All values are based on hierarchical entry step. IPT-M = maintenance interpersonal psychotherapy.

the other nonsevere event categorizations was statistically significant $(p>.05$; see Table 2$)$. The form of the interaction revealedthat subject-focused independent events predicted recurrence for patients receiving medication, whereas the relative absence of such nonsevere events tended to be associated with recurrence for nonmedicated patients. Specifically, for patients receiving medication, $80 \%$ ( 4 of 5 ) patients with a nonsevere event suffered a recurrence, whereas only $16.67 \%$ ( 8 of 48 ) of medicated patients without a nonsevere event experienced a recurrence. For patients not receiving medication, $40 \%$ (4 of 10) with a nonsevere event suffered a recurrence versus $71.43 \%$ (45 of 63) of unmedicated patients without a nonsevere event experiencing a recurrence. ${ }^{9}$ None of the other life event categories interacted with medication status to predict recurrence at the designated alpha level. $^{10}$

Two subsidiary sets of analyses were run to ensure that these findings were robust and independent of other stress effects. First, identical analyses were run, except that they excluded from the sample the 22 dropouts during the Maintenance Phase. ${ }^{11}$ The findings remained essentially the same for nonsevere subjectfocused independent events, $\chi^{2}(1, N=104)=8.92, p<.01$. Second, in a prior study, we found that undesirable life events during the 12-week period preceding the Maintenance Phase also interacted with medication status to predict recurrence (Monroe et al., 1996), with the form of the interaction similar to that indicated for the present findings. Therefore, we controlled for these preMaintenance undesirable events and their interaction with medication status by entering them in a logistic regression analysis; then we entered the hierarchical steps for the nonsevere subjectfocused independent life event and its interaction with medication status. Most importantly, nonsevere subject-focused independent events interacting with medication status continued to significantly predict recurrence above and beyond the previous life event findings, $\chi^{2}(1, N=126)=10.04, p<.002$. Thus, the present results were not influenced by attrition, nor were they attributable to previous effects reported for this sample.

\section{Discussion}

The present findings are consistent with the general premise that nonsevere life events are capable of triggering a recurrence of depression. These data extend the findings of Ormel et al. (2001) by more precisely defining the types of nonsevere life events and by including full syndromal depressive recurrences. The present results, however, are qualified by important and relatively novel considerations. Specifically, the prediction of recurrence by nonsevere life events (a) was primarily for patients receiving medication, (b) involved life events that were focused on the participant, and (c) involved events that were largely outside of his or her control. These findings are of considerable theoretical interest, for they suggest that, with repeated recurrences of depression, progressively less severe forms of life stress can become capable of triggering an episode of depression (Monroe \& Harkness, 2005).

The interaction of life stress with medication treatment is consistent with our prior findings based on a smaller number of patients and a single stress assessment preceding the patient's

\footnotetext{
${ }^{9}$ As noted previously, the 12 -week life stress assessments yielded similar findings to those for the 6-week assessments for the interaction between nonsevere subject focused events and medication status, $\chi^{2}(1, N=$ $126)=13.71, p<.001$, and provide a slightly larger number of patients per cell. For patients receiving medication, $71.42 \%$ (5 of 7) with a nonsevere event suffered a recurrence, whereas $15.22 \%$ (7 of 46) unmedicated patients without a nonsevere event experienced a recurrence. For patients not receiving medication, $43.75 \%$ (7 of 16) with a recent nonsevere life event had a recurrence versus $73.68 \%$ (42 of 57) of unmedicated patients without a recent nonsevere life event experiencing a recurrence.

${ }^{10}$ It should be noted that nonsevere subject-focused probably independent events and nonsevere any focus independent events yielded trends for predicting recurrence in interaction with medication status $(p<.06$ and $p<.09$, respectively).

11 The range of weeks for the 22 dropouts in the Maintenance Phase was 2-95, and the mean number of weeks in the Maintenance Phase was 37.14.
} 
entry into the 3-year follow-up period (Monroe et al., 1996). Whereas the previous results demonstrated the purely prospective prediction of undesirable life events for depression's recurrence in a patient's receiving medication, the present results revealed that life stress continued to predict recurrence when more frequent and sensitive stress measures were used with a greatly expanded sample. Most importantly, the stress prediction based on the present investigation is independent of the stress prediction found in the prior report (i.e., each significantly and independently predicted recurrence). Thus, the present results lend incremental, nonredundant support for the basic prediction of recurrence by nonsevere life stress. In particular, the present results indicate a more temporally proximal triggering role for life events. This suggests that stressors occurring at different times are capable of predicting recurrence in an independent fashion (at least for patients with recurrent depression receiving medication).

Our interpretation of the effects of stress interacting with medication is informed by the specific nature of the present sample of recurrent depressives. By a considerable margin, the patients not receiving medication suffered the most recurrences. Medication thus appears to protect against the extreme stress sensitivity of this very recurrent group of individuals (given the overall much higher rate of recurrence without medication), lowering the liability such that relatively more impactful life events are required to bring about recurrence. Note that, given such findings, we would also expect more severe life events, if sufficient in frequency, to also precipitate recurrence. ${ }^{12}$ The critical question for present purposes, though, is whether particular types of nonsevere life stress can also precipitate the recurrence of depression. Interestingly, these findings regarding life events and medication status may have broader implications for other groups vulnerable to psychopathology. As noted previously, research on medicated and unmedicated schizophrenic patients found stress to figure most prominently in predicting relapse for the medicated schizophrenic patients (Leff et al., 1973). Nonetheless, our prior and present findings of an interaction between life stress and medication are based on the same sample over different times and consequently warrant independent replication.

Why patients not on medication were less likely to recur had they experienced prior stress, however, requires further discussion. One interpretation is that nonsevere stress may serve a protective role with regard to recurrence. For example, people with a long history of depression may monitor their stress, recruit support, seek additional treatment, or increase other coping when acute events happen that could trigger recurrence. Within this viewpoint, stress provides a signal to marshal resources and to increase efforts to prevent recurrence. Slightly different, but also consonant with the viewpoint of stress as affording a protective role, individuals with repeated exposure to nonsevere events over time may develop resilience to adversity and become less vulnerable to depression (e.g., Farmer \& McGuffin, 2003).

Another very different interpretation is also plausible and is based on a vulnerability-stress interaction over time. Whereas recurrent depressives not receiving medication will be highly vulnerable as a group, there will be individual differences in vulnerability. How these differences in vulnerability play out over time in relation to stress is important to consider. Specifically, from a stress sensitization perspective, the most vulnerable individuals will break down with the least amount of stress. Given that lower levels of stress generally have a higher base rate, the lower levels of stress capable of triggering recurrence will predominate in the causal picture. Interestingly, this predominance can lead to an apparent suppression of the more severe forms of stress over time: The highly vulnerable on average recur in response to lower level stressors before the less frequent, more severe, stressors have sufficient time to occur and have an effect (Monroe \& Harkness, 2005). Furthermore, these lower levels of stress may be so relatively minor that they often do not meet definitional criteria for inclusion as an event within the LEDS or other similar systems. In contrast, the least vulnerable individuals from a stress sensitization perspective are less likely to suffer a recurrence overall and are more likely to handle higher levels of stress without breaking down. The net effect of this vulnerability-stress interaction over time parallels nicely these results: The most vulnerable individuals recur with minimal stress (e.g., likely below LEDS threshold for events), and the least vulnerable do not recur while handling higher levels of stress.

Of particular importance, too, is the finding that stress effects are relatively specific to particular categories of experiences (although as we discuss subsequently, there were trends for two of the other categories). The prediction of recurrence for patients on medication was based on subject-focused life events over which the subject had little or no control (i.e., independent events). This indicates that not all nonsevere events are likely to be capable of bringing about a recurrence and that the broad category of nonsevere events is too nonspecific. Without consideration of characteristics such as focus or independence, the predictive strength of life stress for predicting recurrence is diluted. Because only a subset of the life events typically tallied in life event studies may be of consequence for recurrence, this may help explain the relative lack of empirical evidence for nonsevere life stress's predicting the recurrence of depression (see Tables 1 and 2; Monroe \& Hadjiyannakis, 2002; Monroe \& Harkness, 2005).

Although issues pertaining to the independence of life events have been addressed often in the literature, the distinction concerning focus per se has not. Yet, as stressors of lesser threat or severity are assessed and considered, many events are included that are focused primarily on other individuals. For example, severe life events are almost always subject focused (indeed, within the LEDS system, for an event to qualify as severe, the subject must be a central focus of the event). As less severe forms of stress are assessed, however, there will be more events that affect primarily others (e.g., spouse, friend, relative, child, neighbor) in the subject's social sphere. It follows that such events may be of less central psychological relevance to the person (i.e., not subject focused), and thereby less likely to trigger the cognitive and biological mechanisms via which stress may bring about recurrence (Post, 1992; Segal, Williams, Teasdale, \& Gemar, 1995).

\footnotetext{
${ }^{12}$ However, as we have outlined previously (see Monroe \& Harkness, 2005), there is good reason to suspect that, if less severe events become capable of triggering recurrence, these less severe events will become more frequent precipitants of recurrence relative to severe events. Yet the severe events should still possess high impact, should they occur. The relatively low frequency of severe life events preceding recurrences in the present sample is consistent with this line of reasoning.
} 
It is interesting that others have touched on the general issue that we capture with the idea of focus (Shrout et al., 1989; Stueve et al., 1999). For example, in addition to examining the fatefulness of events, Shrout et al. also examined reports of the degree of behavioral disruption entailed by events (based on detailed interview information). They found that events that were classified as major often did not involve much disruption. In particular, events involving deaths ("Close friend died"; "Family member other than spouse or child died") were commonly reported but often did not create much behavioral disruption. This is largely due to the reporting of events of deaths of people who were quite remote from the subject. Once such "overreporting" was taken into account, the predictive power of the "filtered" events was enhanced (Shrout et al., 1989; Stueve et al., 1999). It is probable that there are many lower-level events that also have little central meaning for the subject and that serve to dilute the predicted power of the stress measures.

Although our efforts to detect specific features of nonsevere life events to enhance the prediction of recurrence are promising, much work remains to specify the mechanisms involved and to determine other qualities of life stress that may be depressogenic. For instance, it is noteworthy that similar statistical trends were found for the medication interaction involving nonsevere subject-focused possibly independent events, as well as nonsevere any-focus independent events, predicting recurrence $(p<.06, p<.09$, respectively). With a larger sample or a better characterization of the pathogenic qualities related to such events, it is possible that these events, too, would predict recurrence (Brown, Harris, \& Hepworth, 1995; Kendler, Hettema, Butera, Gardner, \& Prescott, 2003). In contrast, however, it should be emphasized that the most general class of nonsevere life events (i.e., nonsevere any-focus possibly independent events) did not approach statistical significance for predicting recurrence. This latter category corresponds most closely to what other investigators would obtain without taking focus or independence into consideration. Thus, the "focus" and "independence" dimensions represent promising candidates for further research on life stress and the recurrence of depression.

There are potentially significant implications for prevention and treatment. One intriguing implication is that the consequences of stress are more pronounced when individuals are maintained on a treatment regimen of established efficacy to prevent recurrence of depression. This indicates that, during increased periods of mildto-moderate stress for individuals receiving medication, the probability for relapse increases, meriting heightened clinical attention. Although it is premature to make specific recommendations, research on temporary increases in treatment intensity (pharmacological or psychological) would appear warranted. Another line of research suggested by the present study involves targeted psychological procedures to help cope with the mild-to-moderate stressors in the lives of people with recurrent depression receiving maintenance pharmacological treatment. The direction of this work would not be simply to deal with the stressors of everyday life in general but, rather, to focus on the lower level stressors that may have a direct impact on the person and may be outside of his or her personal control. Such ideas may lead to more psychological precision in treatment and prevention development, helping focus and strengthen the science of relapse prevention for major depression (Segal, Pearson, \& Thase, 2003).
Despite the belief that nonsevere life stress is an important element in depression's recurrence, few studies have addressed the matter. None, to our knowledge, has covered a 3-year longitudinal time span, incorporating state-of-the-art stress assessments every 12 weeks. The procedures adopted for defining remission and recurrence in the present research, too, provide a strong methodological platform for examining the study hypotheses. The potential limitations of the work, however, also should be addressed. Despite the high quality of our stress measure and reliability data for one of the primary rating dimensions (long-term threat), we do not have reliability data on the independent versus possibly independent distinction (although such decisions are made with often detailed guidance from the LEDS manual). Another arena involves generalizability issues. Our findings are applicable to patients with a history of recurrent depression. Further work is required to determine whether the findings hold for patients with fewer lifetime episodes, for patients with nonrecurrent, or less recurrent, depression, or for both (Monroe \& Harkness, 2005). The findings, too, are based on patients who have recovered from a particular combination of treatment (i.e., IPT and imipramine), who have sustained their treatment gains, and who continue in the treatment protocol. Further work is required to determine whether (a) patients with recurrent depression receiving other forms of intervention or (b) patients with recurrent depression not receiving treatment evidence similar relations with the different categories of nonsevere life stress.

\section{References}

Abramson, L. Y., Seligman, M. E. P., \& Teasdale, J. (1978). Learned helplessness in humans: Critique and reformulation. Journal of Abnormal Psychology, 87, 49-74.

American Psychiatric Association. (2000). Diagnostic and statistical manual of mental disorders (4th ed., text rev.). Washington, DC: Author.

Angst, J., Kasper, S., \& Weiller, E. (2000). Recurrent brief depression: A frequent syndrome in clinical practice. International Journal of Psychiatry in Clinical Practice, 4, 195-199.

Anisman, H., \& Matherson, K. (2005). Stress, depression, and anhedonia: Caveats concerning animal models. Neurosciences and Biobehavioral Reviews, 29, 525-546.

Beck, A. T., Steer, R. A., \& Garbin, M. G. (1988). Psychometric properties of the Beck Depression Inventory: Twenty-five years of evaluation. Clinical Psychology Review, 8, 77-100.

Brown, G. W., \& Harris, T. O. (1978). Social origins of depression: A study of psychiatric disorder in women. New York: The Free Press.

Brown, G. W., \& Harris, T. O. (1989). Depression. In G. W. Brown \& T. O. Harris (Eds.), Life events and illness (pp. 49-93). London: Guilford Press.

Brown, G. W., Harris, T. O., \& Hepworth, C. (1995). Loss, humiliation and entrapment among women developing depression: A patient and nonpatient comparison. Psychological Medicine, 25, 7-21.

Cohen, J., \& Cohen, P. (1983). Applied multivariate regression/correlation analysis for the behavioral sciences (2nd ed.). Hillsdale, NJ: Erlbaum.

Dohrenwend, B. P., Krasnoff, L., Askenasy, A. R., \& Dohrenwend, B. S. (1978). Exemplification of a method for scaling life events: The PERI Life Events Scale. Journal of Health and Social Behavior, 19, 205-229.

Farmer, A., \& McGuffin, P. (2003). Humiliation, loss and other types of life events and difficulties: A comparison of depressed subjects, healthy controls and their siblings. Psychological Medicine, 33, 1169-1175.

Frank, E., \& Kupfer, D. J. (2003). Progress in the therapy of mood disorders: Scientific support. American Journal of Psychiatry, 160, 1207-1208. 
Frank, E., Kupfer, D. J., Perel, J. M., Cornes, C., Jarrett, D. B., Mallinger, A. G., et al. (1990). Three-year outcomes for maintenance therapies in recurrent depression. Archives of General Psychiatry, 47, 1093-1099.

Gotlib, I. H., \& Hammen, C. L. (Eds.). (2002). Introduction. Handbook of depression. New York: Guilford Press.

Hamilton, M. (1960). A rating scale for depression. Journal of Neurology, Neurosurgery, and Psychiatry, 23, 56-62.

Hammen, C. (2005). Stress and depression. Annual Review of Clinical Psychology, 1, 293-319.

Hlastala, S. A., Frank, E., Kowalski, J., Sherrill, J. T., Tu, X. M., Anderson, B., \& Kupfer, D. J. (2000). Stressful life events, bipolar disorder, and the "kindling model." Journal of Abnormal Psychology, 109, 777-786.

Holmes, T. H., \& Rahe, R. H. (1967). The Social Readjustment Rating Scale. Journal of Psychosomatic Research, 11, 213-218.

Kanai, T., Takeuchi, H., Furukawa, T. A., Yoshimura, R., Imaizumi, T., Kitamura, T., \& Takahashi, K. (2003). Time to recurrence after recovery from major depressive episodes and its predictors. Psychological Medicine, 33, 839-845.

Keller, M. B. (2003). Past, present, and future directions for defining optimal treatment outcome in depression: Remission and beyond. Journal of the American Medical Association, 289, 3152-3160.

Keller, M. B., \& Borland, R. J. (1998). Implications of failing to achieve successful long-term maintenance treatment of recurrent unipolar major depression. Biological Psychiatry, 44, 348-360.

Kendler, K. S., Hettema, J. M., Butera, F., Gardner, C. O., Prescott, C. A. (2003). Life event dimensions of loss, humiliation, entrapment, and danger in the prediction of onsets of major depression and generalized anxiety. Archives of General Psychiatry, 60, 789-796.

Kendler, K. S., Thornton, L. M., \& Gardner, C. O. (2000). Stressful life events and previous episodes in the etiology of major depression in women: An evaluation of the "kindling" hypothesis. American Journal of Psychiatry, 157, 1243-1251.

Kennedy, N., Abbott, R., \& Paykel, E. S. (2003). Remission and recurrence of depression in the maintenance era: Long-term outcome in a Cambridge cohort. Psychological Medicine, 33, 827-838.

Kessler, R. C. (1997). The effects of stressful life events on depression. Annual Review of Psychology, 48, 191-214.

Klerman, G. L., Weissman, M. M., Rounsaville, B. J., \& Chevron, E. S. (1984). Interpersonal psychotherapy of depression. New York: Basic Books.

Lee, A. S. (2003). Better outcomes for depressive disorders? Psychological Medicine, 33, 769-774.

Leff, J. P., Hirsch, S. R., Gaind, R., Rohde, P. D., \& Stevens, B. C. (1973). Life events and maintenance therapy in schizophrenic relapse. British Journal of Psychiatry, 123, 659-660.

Maier, S. F., \& Watkins, L. R. (2005). Stressor controllability and learned helplessness: The roles of the dorsal raphe nucleus, serotonin, and corticotropin-releasing factor. Neurosciences and Biobehavioral Reviews, 29, 829-841.

Mazure, C. M. (1998). Life stressors as risk factors in depression. Clinical Psychology: Science and Practice, 5, 291-313.

McQuaid, J. R., Monroe, S. M., Roberts, J. E., \& Johnson, S. L. (1992). Toward the standardization of life stress assessment: Definitional discrepancies and inconsistencies in methods. Stress Medicine, 8, 47-56.

Monroe, S. M., \& Hadjiyannakis, K. (2002). The social environment and depression: Focusing on severe life stress. In I. H. Gotlib \& C. L.
Hammen (Eds.), Handbook of depression (pp. 314-340). New York: Guilford Press.

Monroe, S. M., \& Harkness, K. L. (2005). Life stress, the "kindling" hypothesis, and the recurrence of depression: Considerations from a life stress perspective. Psychological Review, 112, 417-445.

Monroe, S. M., Kupfer, D., \& Frank, E. (1992). Life stress and treatment course in recurrent depression. I: Response during index episode. Journal of Consulting and Clinical Psychology, 60, 718-724.

Monroe, S. M., \& Roberts, J. R. (1990). Conceptualizing and measuring life stress: Problems, principles, procedures, progress. Stress Medicine, 6, 209-216.

Monroe, S. M., Roberts, J. E., Kupfer, D. J., \& Frank, E. (1996). Life stress and treatment course of recurrent depression: II. Postrecovery associations with attrition, symptom course, and recurrence over 3 years. Journal of Abnormal Psychology, 105, 313-328.

Monroe, S. M., \& Simons, A. D. (1991). Diathesis-stress theories in the context of life stress research: Implications for the depressive disorders. Psychological Bulletin, 110, 406-425.

Ormel, J., Oldehinkel, A. J., \& Brilman, E. I. (2001). The interplay and ecological continuity of neuroticism, difficulties, and life events in the etiology of major and subsyndromal, first and recurrent depressive episodes in later life. American Journal of Psychiatry, 158, 885-891.

Post, R. M. (1992). Transduction of psychosocial stress into the neurobiology of recurrent affective disorder. American Journal of Psychiatry, 149, 999-1010.

Rabkin, J. G., \& Klein, D. F. (1987). The clinical measurement of depressive disorders. In A. J. Marsella, R. M. A. Hirschfeld, \& M. M. Katz (Eds.), The measurement of depression (pp. 30-83). New York: Guilford Press.

Raskin, A., Schulterbrandt, J. G., Reatig, N., \& McKeon, J. J. (1969). Replication of factors of psychopathology in interview, ward behavior and self-report ratings of hospitalized depressives. Journal of Nervous and Mental Diseases, 148, 87-98.

Segal, Z. V., Pearson, J. L., \& Thase, M. E. (2003). Challenges in preventing relapse of major depression: Report of a National Institute of Mental Health Workshop on state of the science of relapse prevention in major depression. Journal of Affective Disorders, 77, 97-108.

Segal, Z. V., Williams, J. M., Teasdale, J. D., \& Gemar, M. (1995). A cognitive science perspective on kindling and episode sensitization in recurrent affective disorder. Psychological Medicine, 26, 371-380.

Shrout, P. E., Link, B. G., Dohrenwend, B. P., Skodol, A. E., Stueve, A., \& Mirotznik, J. (1989). Characterizing life events as risk factors for depression: The role of fateful loss events. Journal of Abnormal Psychology, 98, 460-467.

Spitzer, R. L., Endicott, J., \& Robins, E. (1978). Research diagnostic criteria. Archives of General Psychiatry, 34, 773-782.

Stueve, A., Dohrenwend, B. P., \& Skodol, A. E. (1999). Relationships between stressful life events and episodes of major depression and nonaffective psychotic disorders: Selected results from a New York Risk Factor Study. In B. P. Dohrenwend (Ed.), Adversity, stress, and psychopathology (pp. 341-357). New York: Oxford University Press.

Received January 3, 2005

Revision received September 6, 2005 Accepted September 12, 2005 